\title{
Évolution, corps, langage : le cas Paul Alsberg et l'anthropologie philosophique
}

\section{Mario Marino}

\section{OpenEdition}

\section{Journals}

Édition électronique

URL : http://journals.openedition.org/alter/379

DOI : $10.4000 /$ alter.379

ISSN : 2558-7927

Éditeur :

Association ALTER, Archives Husserl (CNRS-UMR 8547)

\section{Édition imprimée}

Date de publication : 1 novembre 2015

Pagination : 116-131

ISBN : 978-2-9550449-1-9

ISSN : $1249-8947$

\section{Référence électronique}

Mario Marino, «Évolution, corps, langage : le cas Paul Alsberg et l'anthropologie philosophique », Alter [En ligne], 23 | 2015, mis en ligne le 01 décembre 2017, consulté le 09 mai 2019. URL : http:// journals.openedition.org/alter/379; DOI : 10.4000/alter.379 


\section{ÉVOLUTION, CORPS, LANGAGE : LE CAS PAUL ALSBERG ET L'ANTHROPOLOGIE PHILOSOPHIQUE ${ }^{1}$}

Mario Marino

Dans l'histoire et le débat autour de l'anthropologie philosophique allemande du XXe siècle, l'œuvre et le destin du médecin juif-allemand Paul Alsberg (1883-1965) constituent un véritable cas qui permit d'interroger la genèse et la validité de l'anthropologie philosophique même. En suivant Joachim Fischer, on entend ici par "Philosophische Anthropologie » une orientation de la pensée du $\mathrm{XX}$ e siècle, dont le fil rouge offre une réponse originale à la «destruction de l'idéalisme pendant le XIXe siècle $»^{2}$. L'anthropologie philosophique propose une réflexion sur l'esprit humain non plus à partir des « prestations du sujet » mais à partir $d u$ «fait du vivant », autrement dit indirectement. L'anthropologie philosophique tend vers le sujet en se donnant comme point de départ un "pôle objectif », placé avant et au-dessous du sujet même, " entre matière et homme ». Ce pôle est la vie, pensée dans sa corrélation organique avec l'environnement. Ce lien vital devient l'objet d'une enquête comparative révélant une " position particulière » (Sonderstellung) de l'homme dans la nature ${ }^{3}$ : à partir de cette position particulière même, l'anthropologie philosophique pense l'unité et la liberté de la nature humaine et la «fon-

\footnotetext{
${ }^{1}$ P. Alsberg, Das Menschheitsrätsel. Versuch einer prinzipiellen Lösung, Dresde, Sibyllen, 1922, sera cité avec l'abréviation $\mathrm{M}$, immédiatement suivie du numéro de page. Je souhaite exprimer ma gratitude à Étienne Bimbenet pour son invitation au séminaire de la revue Alter et aux participants à la séance du 31 de Janvier 2015 - en particulier à Christian Sommer - pour les questions soulevées au cours du débat. Je dédie ce travail à Caterina Zanfi, à sa générosité contagieuse.

${ }^{2}$ J. Fischer, Philosophische Anthropologie. Eine Denkrichtung des 20. Jahrhunderts, Fribourg, Alber, 2008, p. 511.

${ }^{3}$ Ibid., p. 524.
} 
dation des sciences de la nature et de la culture en tant que formes de la connaissance humaine $»^{4}$.

Si dans les années 1970 un telle approche semblait avoir épuisé sa force argumentative, depuis la fin des années 1980 en Allemagne et la fin des années 1990 elle connaît une renaissance historiographique et théorique remarquable. Un des effets de cette résurgence est l'ouverture d'un dialogue entre l'anthropologie philosophique et d'autres traditions philosophiques telles que le pragmatisme et la phénoménologie. Dans ce contexte, Fischer propose non seulement un élargissement très discuté du canon ${ }^{5}$ (en considérant comme auteurs majeurs, - outre Max Scheler, Helmuth Plessner et Arnold Gehlen - le Kulturphilosoph Erich Rothacker et le biologiste Adolf Portmann) mais il met tout en œuvre pour hisser Alsberg au rang des penseurs éminents de l'anthropologie philosophique ${ }^{6}$. Dans les pages suivantes, j'esquisserai d'abord un cadre de la réception d'Alsberg, puis je fournirai une explication des conditions théorétiques et culturelles de l'anthropologie alsbergienne, à savoir la confrontation avec le darwinisme, la doctrine de la nature biologiquement déficiente de l'homme, la théorie du langage et de la raison. Ce faisant, je m'attacherai plus particulièrement à élucider le rapport entre Gehlen et Alsberg.

\section{Moments de la présence d'Alsberg dans et autour de l'anthropologie philosophique}

Dans l'histoire de l'anthropologie philosophique le nom d'Alsberg émerge par vagues. Il est mentionné une première fois dans l'écrit fondamental de Scheler, "Die Stellung des Menschen im Kosmos", où Alsberg est mentionné et critiqué en tant que représentant d'une anthropologie antispiritualiste de la déficience : «Alsberg refuse expressément de définir l'homme d'abord par l'esprit et la raison » et ce

\footnotetext{
${ }^{4}$ Ibid., p. 526.

${ }^{5} \mathrm{~J}$ 'ai fourni un compte rendu de cette querelle in M. Marino, «Sull'antropologia filosofica come indirizzo di pensiero nella Germania del ventesimo secolo e in prospettiva odierna. A proposito dell'ultimo libro di Joachim Fischer», Intersezioni. Rivista di storia delle idee, $\mathrm{n}^{\circ} 1$, Bologne, Il Mulino, 2011, p. 143-151. Une tentative de réponse par Fischer lui-même à la majorité des critiques se trouve dans l'avant-propos à la deuxième édition de son livre.

${ }^{6}$ Fischer a chargé le sociologue franco-allemand Aldo Haesler de rédiger un chapitre sur Alsberg pour un ouvrage de référence dirigé par Fischer lui-même pour la collection « Philosophische Anthropologie. Themen und Positionen » de l'éditeur Bautz. Voir A. Haesler, «Wurf und Schlag. Paul Alsberg und das Körperausschaltungsprinzip », in Philosophische Anthropologie. Hauptautoren und Grundschriften (à paraître 2015).
} 
faisant, il réduit l'esprit à un "succédané tardivement apparu d'une insuffisance d'adaptation [...], une surcompensation de l'infériorité organique constitutionnelle de l'espèce humaine $»^{7}$. Scheler, au contraire, considère acquis le fait que l'esprit « est un principe opposé à toute vie en général » et «n'est pas réductible à l'évolution naturelle de la vie; s'il se ramène à quelque chose $c^{\prime}$ est seulement au fondement ultime du monde $»^{8}$.

En défenseur d'une anthropologie de la déficience assez productive du point de vue de la philosophie de la technique, Alsberg remporte néanmoins l'adhésion et l'éloge de Gehlen après la deuxième guerre mondiale. Alsberg aurait en effet saisi «le rapport de compensation qui existe entre le déficit biologique dont souffre l'homme $\mathrm{du}$ point de vue des ses organes et l'intelligence qu'il déploie dans son langage et dans ses actes ${ }^{9}{ }^{\text {; }}$ à la suite de cette compensation l'homme développe des techniques, des instruments et des connaissances en s'appuyant sur sa propre expérience et non sur le modèle de la nature. Pour Gehlen, qui présente sa propre anthropologie philosophique comme une actualisation non-darwinienne de l'anthropologie herderienne, l'homme serait un «Mängelwesen » (un être lacunaire), c'est-à-dire un être vivant qui, contrairement à l'animal, ne dispose ni d'organes ni d'instincts spécialisés et par là même il ne possède pas non plus d'économie pulsionnelle régulée a priori. Maîtriser le surplus de stimuli dû à une telle situation, pesant comme une charge sur l'individu, est donc un accomplissement d'une importance vitale : chez Gehlen il s'agit de l'«agir» qui vise l'édification du monde positif de la culture. En outre, l'instrument de "décharge » (Entlastung) et de culture le plus puissant est, selon Gehlen, la langue : les symboles linguistiques sont en effet l'expression concentrée de la relation entre l'homme et l'objet de l'expérience. Ils servent à l'homme pour aller au-delà de la situation particulière, et à travers eux la réalité devient le produit et l'instrument de nouvelles actions humaines.

Interné à Oranienburg en 1933, Alsberg est libéré un an plus tard, grâce à l'intercession de sa femme auprès de l'ambassade britannique, et il émigre aussitôt. De son côté, Gehlen est membre du NSDAP pendant la période nazie. Or voici qu'il se prononce justement, après la guerre, en faveur d'une victime du national-socialisme,

\footnotetext{
${ }^{7}$ M. Scheler, La situation de l'homme dans le monde, traduit et préfacé par M. Dupuy, Paris, Aubier, 1951, p. 78.

${ }^{8}$ M. Scheler, La situation de l'homme dans le monde, op. cit., p. 52-53.

${ }^{9}$ A. Gehlen, Essais d'anthropologie philosophique, Paris, Maison des sciences de l'homme, 2009, p. 123-124.
} 
à savoir Alsberg : cela ne va pas sans déclencher deux polémiques qui prennent cours pendant la seconde moitié du $X X^{\mathrm{e}}$ siècle. Plessner, qui avait été contraint d'émigrer, et Wolfgang Harich, l'un des philosophes les plus fins de la DDR, accusent Gehlen - le premier en l'insinuant, le second en l'affirmant clairement - d'avoir plagié Alsberg qu'il ne mentionne qu'après la défaite du nazisme ${ }^{10}$. Plessner en parti-

culier avance que la catégorie gehlenienne de Entlastung et sa signification systématique pour l'anthropologie ont été préformées par Alsberg ${ }^{11}$. Le soutien au nazisme de Gehlen et son mépris pour la BRD de l'après-guerre ne font aucun doute, mais tant sur le plan théorétique que philologique l'accusation de plagiat est insoutenable. $\mathrm{D}^{\prime}$ une part, les affinités théorétiques entre Gehlen et Alsberg sont dues au fait que les deux auteurs se référent aux mêmes sources, d'autre part, leur cadre théorique est indéniablement divergent: la déficience biologique de l'homme chez Alsberg n'est pas le point de départ, mais un effet de l'évolution technique de l'homme.

La dimension évolutionnaire de l'anthropologie alsbergienne a attiré l'attention notamment de Dieter Claessens et Hans Blumenberg. Dès les années 1960 et jusqu' au début des années 1980'2, Claessens, en théoricien d'une anthropologie sociologique, a utilisé Alsberg comme une sorte de correctif pour les philosophies et les sciences dont l'effort visait une définition de la nature humaine et de la position de l'homme dans le monde. Claessens entend avant tout améliorer l'anthropologie de Gehlen dans deux directions : élargir d'une part l'horizon scientifique de la théorie gehlenienne par l'inclusion des modèles évolutionnistes d'explication du développement humain et d'autre part, neutraliser les tendances réactionnaires et autoritaires de Gehlen, présentes à la fois dans sa doctrine des institutions et dans ses analyses de la société contemporaine. Une telle neutralisation doit s'opérer par un affaiblissement du théorème gehlenien du Mängelwesen. À partir de 1975, Blumenberg utilise à son tour Alsberg contre Gehlen, mais son cadre est l'anthropologisation de la phénoménologie. Toutefois, il est

\footnotetext{
${ }^{10}$ Sur Gehlen et Harich, voir K.-S. Rehberg, Kommunistische und konservative Bejahungen der Institutionen - Eine Brief-Freundschaft, in Wolfgang Harich zum Gedächtnis, Munich, Müller \& Nerding, 2000, vol. 2, p. 440-486.

${ }^{11}$ H. Plessner, Gesammelte Werke Band IV, Berlin, Suhrkamp, p. 25.

12 Voir D. Claessens, Instinkt, Psyche und Geltung, Cologne, Westdeutscher Verlag, 1970, p. 8193 ; Nova Natura, Düsseldorf/Cologne, Diederichs, 1970, p. 12-25 ; Das Konkrete und das Abstrakte, Francfort-sur-le-Main, Suhrkamp, 1980, p. 60-66.
} 
surprenant que Blumenberg, dans son traité inachevé mais presque terminé, ait passé Claessens sous silence ${ }^{13}$.

Cet esquisse très rapide $d^{\prime}$ une histoire de la réception montre deux choses : d'une part, la réception d'Alsberg dans l'anthropologie philosophique peut être désignée comme un malentendu à la fois productif et polarisant et d'autre part, s'il n'est pas facile de ranger les idées alsbergiennes dans l'anthropologie philosophique, on ne peut nier qu'elles y sont étroitement reliées. Il s'agit maintenant de les analyser de plus près.

\section{Entre évolution et intuition : la solution alsbergienne de l'énigme anthropologique}

Alsberg est au fond l'auteur d'une seule œuvre: publié en 1922 à Dresde, brûlé par les nazis en 1933 et republié à Vienne en 1937, ce livre est intitulé Das Menschheitsrätsel. Versuch einer prinzipiellen Lösung. Le terme Menschheitsrätsel est à la fois une allusion claire et une prise de distance d'avec Ernst Haeckel, dont l'ouvrage le plus populaire s'intitulait Die Welträtsel. L'adjectif prinzipiell renvoie quant à lui à la stratégie alsbergienne pour résoudre de manière radicale et définitive le problème anthropologique en introduisant un nouveau principe unitaire. Aussi, toute la pensée d'Alsberg gravite-t-elle autour d'un seul et même principe qu'il nomme Körperausschaltung et interprète comme le principe de définition et, à la fois, d'évolution de l'humanité. L'idée centrale est que soudain et à un moment indéterminé14, le précurseur de l'homme se tient à distance de son ennemi dans la lutte pour l'existence non plus en fuyant, mais en jetant des pierres. Le principe de l'hominisation et de l'humanité est alors cette distanciation $^{15}$ : dans un premier temps, celle-ci a lieu non plus au moyen d'armes naturelles mais d'instruments matériels, ensuite elle se pour-

\footnotetext{
${ }^{13} \mathrm{H}$. Blumenberg, Description de l'homme, Paris, Cerf, 2011.

${ }^{14}$ En articulant de manière très subtile les motifs paléoanthropologiques, phénoménologiques et de l'anthropologie philosophique, Blumenberg a tenté de déterminer un tel passage dans l'histoire évolutionnaire humaine. Aussi l'a-t-il défini comme le résultat d'un processus de transformation très long ayant touché tant les structures de la conscience que les structures morphologiques et les conditions extérieures de l'existence biologique, un processus étroitement lié au changement de biotope de la forêt vierge à la steppe (H. Blumenberg, Description de l'homme, op. cit., p. 513-541).

${ }^{15} \mathrm{La}$ distanciation devient aussi une catégorie anthropologique essentielle pour Claessens (voir par exemple Nova Natura, op. cit., p. 5: «l'histoire de l'humanité est une histoire de distanciation») et Blumenberg (Description de l'homme, op. cit., p. 570 : « eine Antwort auf die Frage, wie der Mensch möglich sei, könnte daher lauten : durch Distanz », " une réponse à la question de savoir comment l'homme est possible pourrait être : par la distance »).
} 
suit et s'intensifie par le biais d'instruments immatériels, à savoir le signe linguistique et le concept. À la différence de Haeckel, pour lequel la pensée évolutionniste fournit une explication universelle et unitaire de la totalité des phénomènes naturels, Alsberg se sert de son principe de manière exclusive pour éclairer le chemin particulier, évolutionnaire de l'homme.

Sur le fond de l'anthropologie alsbergienne, se trouvent aussi deux matrices de l'anthropologie philosophique : la crise de la philosophie traditionnelle face au darwinisme et le débat sur la méthode des sciences naturelles et de la philosophie au tournant du siècle. Alsberg décrit son rapport avec l'évolutionnisme comme prolongement et élargissement original de la pensée évolutionnaire à travers un principe systématique d'espèce intuitive et en résonnance avec la problématique de son temps concernant la définition de la nature humaine. Alsberg n'interroge pas l'évolutionnisme en tant que théorie scientifique, mais la "Weltanschauung » que cette même théorie a mise en évidence, une Weltanschauung qui prive l'homme de toute tension métaphysique - qu'elle soit morale, religieuse, esthétique ou théorétique. Aussi l'homme est-il considéré par l'évolutionnisme seulement comme "espèce animale plus élevée [...] un animal au sens d'un primus inter pares ${ }^{16}$. La conséquence la plus lourde de cette animalisation totale de l'homme est pour Alsberg un " conflit de conscience » (Gewissenskonflikt) dramatique dans l'homme et le savoir humain entre le «sentiment» (Gefühl) et «l'entendement» (Verstand), le «cœur» $($ Herz $)$ et la « raison » (Vernunft) entre la logique du sentiment et celle $\mathrm{du}$ fait, en somme entre philosophie et science naturelle. En exposant ce conflit, Alsberg met au jour deux aspects fondamentaux du senti-

\footnotetext{
${ }^{16} \mathrm{M}, 11$. Telle est aussi la raison pour laquelle, dans le livre d'Alsberg, il est généralement question d'une "réintégration de l'homme dans des droits et prérogatives anciens, dont une exégèse erronée de la doctrine du développement [à savoir : l'évolutionnisme] l'a privé » (ibid.). Sur ce sujet, je renvoie aussi à l'analyse des positions évolutionnistes sur la nature de l'homme et sur son importance par rapport au royaume animal dans la première partie de Menschheitsrätsel, p. 67-71. Alsberg s'insurge contre les incursions - à son avis vouées à l'échecde Darwin dans l'anthropologie : il critique entre autres l'affirmation fatale de Darwin, selon laquelle l'effort pour définir l'essence de l'homme aurait moins d'importance que la recherche empirique sur l'évolution des formes de vie concrètes ayant conduit à la forme humaine actuelle. Quoi qu'il en soit, le ton polémique d'Alsberg vise principalement les développements anthropologiques de la pensée darwinienne en Allemagne, en particulier ceux de Haeckel. En commentant certaines systématiques zoologiques de Haeckel, Alsberg parvient aux conclusions générales suivantes sur les hypothèses anthropologiques de l'évolutionnisme: "L'homme primitif est-il seulement un singe et l'homme d'aujourd'hui est-il alors simplement un singe ? Voilà à quelles absurdités conduit le manque de définition conceptuelle de l'homme, absurdités qui ne sont au final rien d'autre que la conséquence extrême du point de vue moderne de la science naturelle. Ce sont elles, néanmoins, qui illustrent le mieux la conclusion erronée de la science au sujet de l'animalité de l'homme » $(\mathrm{M}, 71)$.
} 
ment. D'une part, le sentiment garantit à l'homme une vocation qui conduit ce dernier au-delà de l'animalité et n'ayant aucune preuve de cette vocation, la science moderne ne fait rien d'autre que la nier. D'autre part, selon Alsberg, le fait que le sentiment soit exclu des moyens dits légitimes de connaissance de la réalité a justement permis à la recherche autour des phénomènes naturels de surmonter un obscurantisme séculaire, de parvenir au statut de science et dès lors, de produire systématiquement des connaissances certaines. Formé en médecine alors que le positivisme est à son apogée, Alsberg est aussi un savant attentif à la sensibilité humaine et il croit profondément dans la fonction salvatrice de la science pour l'humanité entière. Fort d'une telle conviction, Alsberg n'est pas disposé à renoncer aux conquêtes fondamentales de la civilisation du XIX ${ }^{\mathrm{e}}$ siècle. Mais en même temps, il admet le rôle incontournable du sentiment même pour le domaine de la science naturelle - dans l'autoconscience et dans l'orientation de l'homme dans le monde. Selon Alsberg, la science ne peut véritablement accomplir sa mission, à savoir libérer l'humanité de toute peur et inquiétude, que si le sentiment s'accorde avec les vérités scientifiques: "Une conclusion exacte du point de vue logique peut certes être tirée et être, de ce fait, contraignante. Mais sur la justesse de son contenu, c'est bien souvent le sentiment qui tranche, nous libérant lorsqu'il s'accorde avec la conclusion, nous précipitant dans le doute, lorsqu'il s'y oppose ${ }^{17}$.

L'issue (Ausweg) de ce conflit consiste pour Alsberg dans une méthode intuitive appliquée à la philosophie kantienne : si à l'instar du morphologiste de la culture Oswald Spengler, alors au sommet de sa popularité, Alsberg plaide pour une reprise de la procédure intuitive dans la philosophie, il ne se réfère pas à Goethe, comme Spengler, mais à Kant. En outre, la plupart des commentateurs ont négligé ce qui, pourtant, est la description par Alsberg lui-même du sens de son anthropologie : le principe de la mise hors circuit du corps surmonte l'opposition entre sentiment et science par une justification évolutionniste de la vocation humaine à dépasser l'animalité.

Alsberg s'est frayé un chemin vers son principe en renversant biologiquement et anthropologiquement le problème kantien de la possibilité de jugements synthétiques a priori : « À vrai dire, la présence de tels jugements ne signifie rien de moins que l'homme est complètement en mesure d'élargir ses connaissances à travers la raison pure (sans recourir à l'expérience extérieure) ${ }^{18}$. En quittant

\footnotetext{
${ }^{17} \mathrm{M}, 12$.
}

${ }^{18} \mathrm{M}, 13$. 
le champ du moi transcendantal pour entrer dans celui de l'homme en tant qu'être naturel, comment peut-on traduire cette théorie kantienne dans le domaine biologique et physiologique ? Comme suit: l'homme est homme, c'est-à-dire qu'il est capable d'une connaissance pure, abstraite, objective s'il met de côté ce qui est sensible et empirique, autrement dit ses propres organes et son propre corps : ainsi, il s'affranchit de la nature. La mise hors circuit du corps (Körperausschaltung) perçue comme délivrance de la nature devient alors un processus constitutif du principe même de l'humanité : à partir de là, Alsberg retrace l'histoire de l'évolution du genre humain à partir des ancêtres animaux jusqu'à l'homme cultivé du XXe siècle. Je n'entends pas fournir ici un rapport exhaustif de l'ouvrage d'Alsberg. Je souhaite par contre approfondir deux axes : la doctrine de la déficience organique et instinctuelle de l'homme et la théorie de la raison et du langage.

\subsection{L'homme n'est pas un Mängelwesen, il le devient}

L'homme civilisé contemporain entre en scène comme un être physiquement faible, techniquement avancé et spirituellement raffiné, tandis que ses ancêtres animaux apparaissent extrêmement vigoureux, mais dépourvus de culture matérielle et spirituelle élevée. Alsberg discerne ici une " interrelation et une interaction très étroites entre la régression corporelle et la croissance technique-spirituelle ${ }^{19}$, qui entérinerait le principe anthropologique de la Körperausschaltung comme principe exclusif de l'évolution de l'humanité.

Pour vérifier cette hypothèse de travail, Alsberg indique deux voies: on peut rechercher la présence et l'effet de ce principe soit dans le spirituel d'abord et ensuite dans le corporel ou, inversement, d'abord dans le corporel et ensuite dans le spirituel. La première méthode a été appliquée à plusieurs reprises dans l'histoire de la pensée occidentale, mais elle aurait échoué à cause tant de l'insuffisance des connaissances sur la vie intérieure des animaux que de la complexité plus grande de la vie spirituelle par rapport à la vie corporelle. En outre, Alsberg considère que la deuxième méthode est prometteuse pour deux raisons : " les rapports dans le corporel [sont] beaucoup plus simples et évidents que dans le spirituel » et d'autre part, les différences mises au jour par l'anatomie comparée entre corporalité humaine et corporalité animale sont beaucoup plus nettes

${ }^{19} \mathrm{M}, 95$. 
et immédiatement utilisables dans la recherche empirique que les différences soulignées dans le champ spiritualiste ${ }^{20}$.

Cette alternative méthodologique, pour ainsi dire entre une voie $\mathrm{du}$ bas vers le haut et une voie du haut vers le bas, correspond à peu de choses près à la subdivision alsbergienne de la recherche anthropologique du XIX ${ }^{\mathrm{e}}$ siècle en deux courants majeurs. Le premier est composé, aux côtés de certains outsiders comme Ludwig Geiger et Ludwig Noiré, d'éminents naturalistes de l'époque comme Hugo Obermaier et Erich Wasmann, et de philosophes comme Kapp et le professeur de Scheler, Rudolf Eucken. Ce groupe partage la conviction que la vie spirituelle humaine, centrée sur le privilège de la raison, constitue la différence essentielle entre l'homme et le domaine animal : de ce fait, les formes plus élevées telles que la culture et le savoir (la morale, la religion et les sciences) ainsi que le langage et la technique sont-elles rattachées à la vie spirituelle. D'un point de vue philosophique, Alsberg n'approuve pas une telle option, mais il y a également, à mon sens, une raison idéologique pour laquelle Alsberg critique ce type d'anthropologie: en réalité, chez presque tous ces auteurs (Obermeier était un frère lai, Wassman un jésuite et interprète catholique du darwinisme, Eucken co-fondateur de la Luther-Gesellschaft) le primat du spirituel sur le corporel a un caractère religieux. L'autre courant, majoritaire dans la science naturelle de la fin du XIX ${ }^{\text {e }}$ siècle, est l'évolutionnisme : selon Alsberg, la thèse évolutionniste, en affirmant que l'homme descend du singe, ne fait aucune différence essentielle entre l'homme et l'animal. D'où le terme utilisé par Alsberg même qui rebaptise cette théorie Zooismus. Alsberg ne s'identifie totalement ni avec les spiritualistes ni avec les évolutionnistes: il se rattache à la théorie de l'évolution sans pour autant aboutir à une animalisation complète de l'homme, il rejette la théorie de l'origine spirituelle et rationnelle du langage et de la technique mais il utilise quand même certaines intuitions de la philosophie du langage de Geiger et de Noiré.

Alsberg loue notamment Darwin pour avoir pris le corps comme point de départ dans l'enquête sur la différence entre les privilèges de l'animal et ceux de l'homme. De cette comparaison anatomique, il résulte que l'homme ne présente aucun des privilèges organiques et instinctuels relevés par Darwin chez les animaux :

Il n'a ni un corps en mesure de se défendre, avec lequel il pourrait s'opposer à l'ennemi, ni un degré de vitesse et d'habileté tel qu'il

${ }^{20} \mathrm{M}, 97$. 
pourrait fuir; il ne dispose pas de fins organes sensoriels pour s'apercevoir rapidement d'un ennemi ou d'une proie, et il n'a pas non plus été doté d'une grande fécondité pour rendre possible, même dans des conditions si défavorables, la subsistance de l'espèce ; ni même son corps est-il protégé contre les intempéries, ni sa dentition comme son appareil digestif sont-ils appropriés pour la nourriture brute offerte par la nature [...]. En bref: il manque au corps humain toutes les garanties nécessaires à son existence et à la conservation de son espèce, garanties dispensées à l'animal et sur lesquelles ce dernier peut s'appuyer avec confiance ${ }^{21}$.

Dans ce passage, tout semble indiquer l'homme comme l'être précisément lacunaire que, plus tard, Gehlen rendit populaire. Ce passage est en fait décisif pour la réception d'Alsberg chez Scheler et Gehlen: sans citer la page, Scheler fait écho à ces lignes lorsqu'il résume la position alsbergienne sur l'insuffisance biologique humaine $^{22}$. Quant à Gehlen, dans ses esquisses de la philosophie d'Alsberg, il ne produira qu'une copie du propos schélérien sur ce point précis $^{23}$. Mais Alsberg refuse l'hypothèse de l'être lacunaire qu'il considère biologiquement insoutenable : en effet, anticipant une critique souvent adressée à Gehlen, il note qu'un animal dépourvu d'instincts et d'un équipement organique adéquats ne peut satisfaire l'exigence de l'autoconservation ${ }^{24}$. Selon Alsberg, c'est précisément le concept de lutte pour la vie qui oblige à affirmer que les ancêtres de l'homme ou encore d'autres animaux possédaient des organes naturels pour se conserver et se défendre. Pour Alsberg, la décadence biologique successive de l'homme ne peut donc s'expliquer que de la manière suivante : les organes ont perdu en force et en importance à cause de l'entrée en jeu d'un principe plus efficace du corps et alternatif au corps même.

Ce processus reçoit chez Alsberg diverses appellations telles que Verkümmerung ( a atrophie »), Rückbildung ( régression»), ou encore sekundäres Absinken («affaissement secondaire») des organes physiques. Un tel processus est la conséquence de l'emploi soudain d'un

\footnotetext{
${ }^{21} \mathrm{M}, 98$.

${ }^{22}$ M. Scheler, La situation de l'homme dans le monde, op. cit., p. 47.

${ }^{23}$ A. Gehlen, "Anthropologie philosophique », in Gesamtausgabe, vol. 4, Francfort-sur-le-Main, Klostermann, 1983, p. 238.

${ }^{24}$ M, 98-99 : "Le misérable équipement physique de l'homme est pour ainsi dire un état non naturel et même totalement impensable chez un animal. Un animal traité avec une telle négligence par la nature [ein derartig stiefmütterlich von der Natur behandeltes Tier] serait voué à une mort immédiate. C'est pourquoi les précurseurs animaux de l'homme ne peuvent avoir été constitués de la même façon que l'homme actuel. Ils doivent plutôt avoir bénéficié de la possession d'excellents dispositifs d'adaptation corporels. Sans quoi ils auraient été d'emblée privés de toute possibilité d'existence et de développement et ainsi de la production du genre humain ».
} 
instrument dans la lutte pour l'existence : dès cet instant, l'évolution de l'homme se sépare une fois pour toutes de l'évolution animale. Cet instrument provoque deux effets décisifs et reliés l'un à l'autre : un déclassement des mécanismes naturels de défense et une interruption $\mathrm{du}$ processus d'adaptation physique animale à l'environnement. Le tournant extracorporel dû à la survenue de l'instrument artificiel dans l'évolution se prolonge et se renforce avec l'apparition des instruments immatériels. Donc, si l'homme actuel apparaît biologiquement lacunaire, ce n'est pas la nature qui est responsable ${ }^{25}$, mais le principe qui régit son évolution et définit l'essence humaine comme posée au-delà de frontières insurmontables pour les animaux ${ }^{26}$. En même temps, ce résultat est indissociable de la supériorité technique et spirituelle de l'homme dans la mesure où le même principe est également responsable du progrès et de la régression physique : pour mieux le dire, le progrès est obtenu par la régression.

Ce rapport inversement proportionel entre les degrés de civilisation humaine et les degrés divers d'adaptation physique du vivant à des niveaux différents de l'évolution peut produire de terribles implications sur le plan éthique, ce que les commentateurs d'Alsberg ont laissé dans l'ombre. En effet, il y a là un thème raciste : animé par le même principe avec lequel Alsberg entend résoudre l'énigme de l'humanité, il ne peut être liquidé comme simple héritage du racisme pseudo-scientifique de la fin du $\mathrm{XIX}^{\mathrm{e}}$ siècle. Les peuples primitifs habitant la terre au début du $X^{\mathrm{e}}$ siècle sont explicitement définis par Alsberg comme "races humaines inférieures » (niederen Menschenrassen) : ils sont rangés au plus bas de l'échelle présumée de l'évolution humaine puisque cette échelle est construite par Alsberg selon le principe de l'évolution technique matérielle et immatérielle ${ }^{27}$. Le clivage entre les degrés extrêmes de l'évolution humaine est attesté, selon Alsberg, par les restes paléoanthropologiques dont l'observa-

\footnotetext{
${ }^{25}$ À cet égard, il ne pourrait y avoir de différence plus grande entre les deux auteurs, car pour Gehlen « les lacunes comme les particularités des organes de l'homme doivent être considérées suivant l'idée directrice du non-spécialisé et sont donc, pour le dire positivement, des primitivismes » (A. Gehlen, Gesamtausgabe, vol. 3, Francfort-sur-le-Main, Klostermann, 1990, p. 95). Cela signifie que l'homme n'est pas devenu lacunaire à partir de la dégradation d'une spécificité organique, mais que, dès l'origine, il possédait les traits morphologiques contenus dans sa nature lacunaire. Alsberg est en revanche catégorique en affirmant que " l'atrophie du corps $[\ldots]$ est une conséquence immédiate de sa mise hors circuit par l'outil artificiel » $(M, 102)$.

${ }^{26} \mathrm{M}, 103$ : « l'animal, avec son corps, est remarquablement adapté aux conditions de vie, tandis que l'homme a porté son corps dans un état [...] d'inaptitude et d'indigence (Untüchtigkeit und Bedürftigkeit) [...]. Le principe du développement de l'animal est celui de l'adaptation du corps (formation ultérieure du corps), le principe du développement de l'homme est la mise hors circuit du corps par le biais d'outils artificiels ».

${ }^{27} \mathrm{M}, 99$.
} 
tion montre que plus l'homme s'éloigne des degrés les plus élevés dans son histoire culturelle, plus il se rapproche de l'animal.

2.3. Les outils immatériels : langage et raison chez Alsberg et Schopenhauer

Alsberg aborde le problème du langage et de la raison de manière analogue à la question de la déficience organique et instinctuelle : il part de la situation présente de l'objet de sa recherche, en interprétant cette situation non comme reflet de l'être originaire, mais comme produit d'un processus d'évolution. Que les mots d'une langue bien formée expriment des concepts et que les langues bien développées soient éminemment abstraites, cela ne signifie pas pour autant, selon Alsberg, que le langage soit dès son origine un instrument au service de la raison pour donner forme et visibilité aux représentations spécifiques de la raison même. Le seul fait que les langues des peu-ples primitifs soient pauvres en abstractions devrait, en revanche, laisser supposer qu'abstraction et concept doivent être considérés comme des produits assez tardifs d'une évolution. Pour consolider cette hypothèse, Alsberg recourt explicitement à la théorie de la connaissance schopenhauerienne. Scheler avait déjà relevé l'influence de Schopenhauer chez Alsberg et Claessens la documentait ultérieurement. Néanmoins, il me semble qu'au sujet de cette influence, trois éléments importants ont échappé à l'attention de Scheler comme de Claessens: tout d'abord, Alsberg développe la théorie schopenhauerienne de la raison et de l'intellect dans une théorie du langage diamétralement opposée à celle de Schopenhauer ; en outre, Alsberg appuie sa théorie en référence à un auteur déjà mentionné et auquel Gehlen reviendra plus tard dans le cadre de sa doctrine de l'origine du langage: Geiger ${ }^{28}$; et enfin, l'anthropologie linguistique de Herder n'a pas été sans effet sur Alsberg en passant justement par Schopenhauer. On peut donc dire que les mêmes sources ont inspiré aussi bien Gehlen que Alsberg : Herder, Schopenhauer et Geiger.

Schopenhauer aurait défini le Verstand comme une capacité de rendre reconnaissables et signifiantes les sensations en les « condensant » ou les «métamorphosant » dans des représentations intuitives, c'est-

\footnotetext{
${ }^{28}$ Sur les sources de la théorie gehlenienne de l'origine du langage et sa fonction systématique dans la construction d'une anthropologie philosophique anti-spiritualiste, voir M. Marino, "L'anthropologie de la "créature déficiente" [Mängelwesen] et la question de l'origine des langues : chemins de Gehlen vers Herder ", Revue germanique internationale, no 10, en ligne, 2009, p. 187-209.
} 
à-dire individuelles et concrètes. Il s'agirait en somme d'une faculté de l'intuition. Sur cette base fournie par l'intellect, la raison construit des relations parmi les objets ou les représentations en dépassant précisément le concret et l'individuel propres aux représentations intuitives: pour ce faire, la raison extrait de toutes les représentations qu'elle a prises en considération ce qui leur est commun. L'essence de la raison est donc l'abstraction, et l'espèce correspondante de représentation est le concept ${ }^{29}$. Tel est exactement le point du raisonnement où Alsberg opère une combinaison originale entre la théorie schopenhauerienne de la connaissance et la philosophie du langage de Geiger : contrairement à Schopenhauer, pour qui raison et langage se correspondaient, Alsberg associe le langage en premier lieu avec $l^{\prime}$ intuition et, par voie de conséquence, avec l'intellect ${ }^{30}$. Ce faisant, il conçoit alors le couple raison-langage non plus comme unité originaire, mais encore une fois, ce qui est caractéristique de sa pensée, comme point d'arrivée d'un processus évolutionnaire, dans lequel pour reprendre la formule de Geiger - «le concept naît à travers le mot (der Begriff entsteht durch das Wort) ${ }^{31}$. Alsberg utilise l'argument $\mathrm{du}$ caractère concret de l'intuition face au caractère abstrait du concept : le concept comprend de nombreux objets en substituant par abstraction ce qui est commun à plusieurs objets. Par contre, « chaque mot du langage représente à chaque fois un objet concret déterminé, $\mathrm{s}^{\prime}$ érige en représentant de cet objet ${ }^{32}$. Si le mot précède le concept et

\footnotetext{
${ }^{29} \mathrm{M}, 140$.

${ }^{30}$ Une discussion partiellement implicite des thèses de Schopenhauer marque aussi les positions alsbergiennes sur le cerveau et sur la faculté de juger. Alsberg soutient la thèse que le cerveau est l'organe principal de l'entendement et sa dimension actuelle dans l'homme doit donc être expliquée d'une façon évolutionniste comme la conséquence de l'évolution du langage. Une telle affirmation est en tous points opposée à la position de Schopenhauer qui considérait le cerveau humain comme l'organe de la raison. Si le cerveau humain est plus grand que celui des animaux, c'est bien - nous dit-il - parce qu'il héberge la raison, à savoir les représentations abstraites, lesquelles manquent totalement aux animaux. Alsberg mentionne explicitement Schopenhauer à propos de la faculté de juger à laquelle ce dernier attribuait une fonction d'intermédiation en l'entendement et la raison. Cette doctrine, justement perçue par Alsberg comme un effet de l'influence de Kant sur Schopenhauer, ne lui convient pourtant pas : le médecin juif-allemand la juge superflue car ce que Schopenhauer nomme «jugement» (Urteil), serait en fait la réflexion proprement dite, en somme une opération qui est déjà contenue dans l'abstraction.

${ }^{31} C^{\prime}$ est Alsberg même qui suggère une interprétation de sa propre pensée, lorsque peu avant la fin du chapitre 13, il livre un bilan des résultats obtenus jusqu'alors (voir M, p. 154-155) : «Que la création du langage ne soit pas attribuable à la raison, cela ne fait plus aucun doute si l'on en croit les résultats de la recherche linguistique moderne. En effet, on a pu constater en toute certitude que le caractère originaire du langage était intuitif. Par conséquent, le langage est préexistant à la raison et dans son développement, l'homme n'a pas pris le chemin de l'abstrait vers le concret, mais inversement du concret vers l'abstrait (Geiger) ».

${ }^{32} \mathrm{M}, 129$. Pour Alsberg la conceptualisation est - comme Claessens l'avait déjà remarqué - identique à l'abstraction, laquelle est de nouveau conçue comme « une seule opération spirituelle »
} 
si tous deux produisent un rapport avec les objets dans la mesure où mot et concept substituent les perceptions nommées et conçues ${ }^{33}$, cela implique deux choses pour la théorie d'Alsberg : d'une part, mot et concept sont deux types de Körperausschaltung, qui opèrent de manières différentes et à différents niveaux sur une seule et même partie $\mathrm{du}$ corps, à savoir sur les organes sensoriels en tant que moyens de représentation sensible; d'autre part, cette Körperausschaltung, qui au début du processus évolutionnaire était opérée par des instruments matériels, se prolonge dans un premier temps par les signes linguistiques et, dans un second temps, par le concept ${ }^{34}$. La liberté psychologique, cognitive et communicative issue du processus global de Körperausschaltung par les instruments matériels et immatériels, est à la fois la clé et l'expression d'une autonomie complète par rapport au corps qui constitue le point culminant de l'évolution naturelle de l'homme. Alsberg voit dans cette liberté non seulement une maîtrise du champ de l'expérience intérieure et extérieure mais aussi un accroissement de puissance et le perfectionnement de la capacité de communication et de représentation. Enfin, elle est une nouvelle configuration des motifs de conduite de la vie.

Alsberg développe sa propre théorie en utilisant soit les mêmes passages de Schopenhauer aui inspireront plus tard Gehlen, soit la notion de Besonnenheit (traduit d'habitude par « réflexion » ou «circonspection ») qui est au cœur de l'anthropologie et de la philosophie du langage herderiennes. Schopenhauer mettait déjà en relief la Besonnenheit sans laquelle Gehlen n'aurait certainement pas reconnu en Herder le prédécesseur de sa propre anthropologie. Autrement dit, les similitudes reliant Alsberg à Gehlen relèvent principalement du fait que l'un et l'autre se laissent inspirer par les mêmes auteurs, tandis que leurs divergences se manifestent dans leurs positions opposées face à l'évolutionnisme. Alsberg loue Schopenhauer pour sa définition de l'abstraction, à savoir : « élimination de tous les bagages inutiles [...], on y abandonne ainsi nombre d'éléments inessentiels qui ne faisaient qu'introduire de la confusion dans les choses réelles et l'on opère qu'avec quelques déterminations cependant essentielles,

\footnotetext{
(einzige geistige Operation). La subdivision intellectualiste de la conceptualisation en " trois actions chronologiquement successives" (drei zeitlich aufeinanderfolgende Handlungen), (comparatio, abstractio, reflexio), est donc, selon Alsberg, artificieuse et fausse. Je renvoie aux pages 145147 et pour une synthèse à la page 145 .

${ }^{33}$ Concernant le mot, voir M. p. 129, quant au concept, se reporter au moins à p. 148.

${ }^{34}$ Pour plus de détails, voir p. 129.
} 
pensées in abstracto $»^{35}$. En la commentant, Alsberg entend entériner sa propre doctrine anthropologique, mais en fait son propos résume des autres pensées de Schopenhauer: "Grâce à l'introduction de l'abstractum, le langage est ainsi devenu l'instrument achevé de la communication: chaque chose et chaque événement, chaque situation et chaque relation, proche ou lointain(e), qu'il s'agisse du passé ou du présent, est saisi par le langage et porté vers une représentation mentale ${ }^{36}$. Lorsque Gehlen reprend vingt ans plus tard les mêmes pensées, il analyse et mentionne explicitement Schopenhauer ${ }^{37}$. Quant à Alsberg, voici sa conclusion : «Ainsi le principe de mise hors circuit dans la langue cultivée moderne a atteint le même sommet que dans la technique moderne ${ }^{38}$. Le déploiement ultérieur de cette pensée, dans le chapitre sur l'importance et la particularité du troisième et plus haut niveau de la Körperausschaltung - le niveau de la raison et des concepts -, englobe la sphère du vital et du pratique et il est scellé par la catégorie de Besonnenheit:

[Le concept] attire dans notre domaine de représentations non seulement l'aujourd'hui, mais aussi l'hier et le demain; non seulement ce qui est proche, mais aussi ce qui est loin ; non seulement le singulier, mais aussi le tout et de cette manière il crée un nouveau lien vital qui pénètre de toutes parts. Les motifs intuitifs (liés au présent devant nous et aux représentations intuitives) sont remplacés par des motifs abstraits, lesquels acquièrent une influence dominante sur notre conduite pratique de la vie, en la modifiant en profondeur. Il s'agit de la «Besonnenheit », laquelle caractérise et distingue la vie de l'homme cultivé et lui confère une empreinte supérieure ${ }^{39}$.

Alsberg nomme donc Besonnenheit la totalité de la liberté acquise par l'homme à travers l'exclusion matérielle et immatérielle du corps. Aussi revendique-t-il à la fin de son ouvrage la nature "évolutionniste »- $c^{\prime}$ est à dire que les objets qu'il examine sont considérés comme un «événement naturel de l'évolution » (natürliches Entwicklungsereignis) - de son interprétation. Cette dernière touche à la fois l'ancrage linguistique des concepts, le devenir toujours plus abstrait des langues et aussi toute l'histoire et l'essence proprement dite de

\footnotetext{
${ }^{35}$ M, 134. Voir A. Schopenhauer, Le monde comme volonté et représentation II, Paris, Gallimard, p. 1238.

${ }^{36} \mathrm{M}, 134$

${ }^{37}$ Voir A. Gehlen, « Das Problem des Sprachursprungs », Forschungen und Fortschritte. Nachrichtenblätter der deutschen Wissenschaft und Technik, no 14, 1938, p. 293 (voir également Gehlen, Gesamtausgabe, vol. 3, op. cit., p. 50-52, et un de ses essais les plus remarquables : «Die Resultate Schopenhauer » (A. Gehlen, Gesamtausgabe, vol. 4, op. cit., p. 25-49, surtout p. 34-36).

${ }^{38} \mathrm{M}, 134$.

${ }^{39}$ M, 157-158.
} 
l'homme. Si Gehlen ne partage pas la perspective évolutionniste alsbergienne, il est néanmoins capable d'intégrer d'une façon systématique les mêmes thèmes qui l'ont inspirée - la critique schopenhauerienne de l'idéalisme, la philosophie anti-intellectualiste du langage de Geiger et l'anthropologie linguistique de Herder - dans une anthropologie de la déficience humaine. 